\title{
Comparison with tube core and Magill forceps for nasotracheal intubation: a randomized controlled trial
}

\author{
Rui $\mathrm{Hu}^{1}$, Jingyi $\mathrm{Niu}^{1}$, Lining $\mathrm{Wu}^{1}$, Hao $\mathrm{Sun}^{1}$, Peng Sun ${ }^{1}$, Jiaying Huang ${ }^{1}$, and Junma $\mathrm{Yu}^{1}$ \\ ${ }^{1}$ Anhui Medical University Third Affiliated Hospital
}

November 26, 2020

\begin{abstract}
Objective: Magill forceps are frequently used to complete the process of nasotracheal intubation (NTI). We aimed to identify a tube core that could facilitate the NTI process conveniently without Magill forceps. Methods: Sixty patients scheduled for oral and maxillofacial surgeries with no differences between the two groups (30 per group) with regard to demographic data were enrolled in our study. In Group M, the wire-reinforced endotracheal catheter was inserted into the trachea using Magill forceps. However, in Group T, a tube core bent to the physiological curve of the nasal cavity lubricated with aseptic paraffin oil was inserted into the endotracheal catheter, and it was then withdrawn after the endotracheal catheter was advanced through the glottis under direct vision. Results: All NTIs were completed successfully, and no Magill forceps were used in Group T. There was a significant difference in total NTI time between the two groups (Group M, 59.7 (5.1) sec vs Group T, 52.4 (3.1) sec). Mild epistaxis was observed in 6 patients in Group M and 5 patients in Group T (6/30 vs 5/30, respectively). There was no presence of damaged oral tissue or damage to the teeth in either group. Conclusion: We conclude that using the tube core, which is a disposable sterilised stylet, for NTI is a convenient choice.
\end{abstract}

\section{Title page}

Comparison with tube core and Magill forceps for nasotracheal intubation: a randomized controlled trial

Rui Hu, MD ${ }^{1}$, Jingyi Niu, MD ${ }^{1}$, Lining Wu, MD ${ }^{1}$, Hao Sun, MD ${ }^{1}$, Peng Sun, MD ${ }^{1}$, Jiaying Huang, MD ${ }^{2}$, Junma $\mathrm{Yu}, \mathrm{MD}^{1}$

${ }^{1}$ Department of Anesthesiology, The Third Affiliated Hospital of Anhui Medical University (The First People's Hospital of Hefei), Huaihe Road 390, Hefei 230061, China.

${ }^{2}$ Department of Otorhinolaryngology Surgery, The Third Affiliated Hospital of Anhui Medical University (The First People's Hospital of Hefei), Huaihe Road 390, Hefei 230061, China.

These authors contributed equally: Rui Hu and Jingyi Niu.

Corresponding author: Junma Yu. MD, Department of Anesthesiology, The Third Affiliated Hospital of Anhui Medical University (The First People's Hospital of Hefei), Huaihe Road 390, Hefei 230061, China. Telephone: +8655162183386, E-mail: majuny163@163.com.

Tel.: +8655162183386

Fax: +8655162183448

Financial Support : None

Conflicts of interest : None 
Tel: +8655162183386

Fax: +8655162183448

Keywords: Nasotracheal intubation, Magill forceps, Airway management.

The Data Availability Statement

The data that support the findings of this study are available from the corresponding author, [J.M. Yu], upon reasonable request.

\section{Conflict of interest statement}

The authors declared that they have no conflicts of interest to this work.

Comparison with tube core and Magill forceps for nasotracheal intubation: a randomized controlled trial

\section{INTRODUCTION}

Nasotracheal intubation (NTI) is widely used in the clinic for oral and maxillofacial surgeries. Magill forceps have been frequently used to complete the process of NTI in several previous studies [1-4]. However, this instrument may cause rupture of the cuff or mucosal injury and even lead to infection [5-7].

Softer tracheal tubes, such as reinforced tubes, tend to move along the posterior pharyngeal wall rather than towards the laryngeal inlet, and it is difficult to navigate them into the vocal cord without using Magill forceps [8]. It was reported that a rigid wire tube body bent along the radian of the nasal cavity could help the anaesthesiologist complete NTI [9] more easily. In another study, the use of a stylet resulted in significantly higher first-attempt intubation success among patients undergoing endotracheal intubation compared with the use of a bougie [10]. The tube core (Fig. 1, A) in Fig. 1 D, a malleable rigid stylet, was similar to the wire tube body in the above research [9]. Thus, we hypothesized that a tube core could facilitate the NTI process conveniently without the use of Magill forceps, and using the tube core for NTI is a convenient choice.

\section{METHODS}

This study protocol was approved by the Research Ethics Board of The First People's Hospital of Hefei (No. 2019-12), and patient enrolment was conducted after being registered in the Chinese Clinical Trial Registry (www.Chictr.org.cn, ChiCTR1900027387). Written informed consent was obtained from all patients, and the study was conducted in accordance with the Declaration of Helsinki.

We excluded patients from our study if they fell into any of the following categories: age $<18$ years or $>$ 80 years; a body mass index (BMI) was over $30 \mathrm{~kg} / \mathrm{m}^{2}$; the Cormack and Lehane (CL) grading scale of the laryngoscopic view of 3 or 4 [11]; receiving anticoagulant therapy; a history of nasal deformity (e.g., nasal trauma, surgery, obstruction, and polyps); the presence of maxillofacial cancers; difficulty anticipated in airway management; mentally ill people; and cervical instability. Standard monitoring equipment was used in the operating room, and none of the study subjects were premedicated. All patients were randomized using computer-generated random numbers, and envelopes containing randomization numbers were divided into 2 groups ( $\mathrm{n}=30$ per group) according to the different equipment that would be used to guide NTI: Magill forceps group (Group M) and tube core group (Group T).

An otorhinolaryngologist of our hospital who was blinded to the group assignment used a nasal speculum to check for deformities inside the nostrils and to select the smoother nostril. If the patency of both nostrils was equal, NTI was performed in the right nostril [3]. All patients received the same general anaesthesia with $0.3 \mu \mathrm{g} / \mathrm{kg}$ sufentanil and $1.5-2 \mathrm{mg} / \mathrm{kg}$ propofol intravenously, followed by muscle followed by $0.15 \mathrm{mg} / \mathrm{kg}$ cisatracurium. Manual ventilation was performed with $100 \%$ oxygen through a facemask for 3 min before intubation. The selected nostril was packed with gauze containing epinephrine to prevent bleeding. The $6.5-\mathrm{mm}$ and $6.0-\mathrm{mm}$ wire-reinforced tracheal tubes were used in males and females, respectively (TUORen 
Medical Equipment Co., Henan, China. Fig. 1, B). Anaesthesia was maintained with propofol, remifentanil, sevoflurane and cisatracurium.

An aseptic suction catheter (OD - $4.0 \mathrm{~mm}$, Fig. 1, C) lubricated with liquid paraffin was inserted through the tracheal tube (Fig. 1, B) with its tip protruding approximately $15 \mathrm{~cm}$, and the tube was then advanced through the nasopharynx. The Macintosh laryngoscope was then placed into the patient's mouth. Then, in Group M, the tracheal tube was inserted into the trachea using a conventional technique. However, in Group T, a tube core bent to the physiological curve of the nasal cavity lubricated with aseptic liquid paraffin was inserted into the tracheal tube, and the tube core was then withdrawn after the tracheal tube was advanced through the glottis under direct vision. NTI of all patients was performed, if required, using Magill forceps. The entire process of intubation is shown in Fig. 2 for Group T. The process was performed by an anaesthesiologist who had extensive experience. Then, minute adjustments to ventilation were performed to maintain end-tidal carbon dioxide partial pressure at 35 - $45 \mathrm{mmHg}$ during the whole operation.

The total NTI time, which was defined as the period from when the operator picked up the device to when three successive end-tidal carbon dioxide waves were obtained following intubation [12], was recorded. An observer blinded to the group assignments assessed epistaxis bleeding using direct laryngoscopy after five minutes of completing NTI, and it was scored as one of four grades according to the following modified criteria: no epistaxis (no blood observed on either the surface of the tube or the posterior pharyngeal wall); mild epistaxis (blood apparent on the surface of the tube or posterior pharyngeal wall); moderate epistaxis (pooling of blood on the posterior pharyngeal wall); and severe epistaxis (a large amount of blood in the pharynx that impeded NTI and necessitated urgent orotracheal intubation) [13]. The frequency of using Magill forceps in the two groups was also recorded.

After the end of surgery, neostigmine $1 \mathrm{mg}$ and atropine $0.5 \mathrm{mg}$ were used to reverse the neuromuscular blockade, and the tracheal tube was extubated when the patient was awake. A visual analogue scale (VAS) according to a $10-\mathrm{cm}$ vertical score ranging from $0=$ no pain to $10=$ worst pain imaginable was recorded by an investigator who was blinded to the research at the following time points: $15 \mathrm{~min}, 1 \mathrm{~h}$, and $24 \mathrm{~h}$ after extubation.

To calculate the sample size, we undertook a pilot study with 10 patients in each group (total 20 patients). The NTI time was significantly longer in the Magill forceps group than in the tube core group (Group M, 59.8 (5.1) sec vs Group T vs 53.1 (3.0) sec). For this study, the total sample size to achieve 0.95 power and an $\alpha$-error of 0.05 was 12 patients per group according to $\mathrm{G}^{*}$ Power 3.1.9.4 software. Sixty adult patients who were rated as American Society of Anaesthesiologists (ASA) I or $\Pi$ and whose condition required NTI under general anaesthesia were selected.

\section{Statistical analysis}

Data are expressed as the mean \pm standard deviation. Parametric data were compared between the groups by analysis of variance and post hoc testing. Categorical data were analysed using Fisher's exact test. Statistical significance was considered at $P$ values $<0.05$. All statistical analyses were performed with Statistical Package for Social Sciences (SPSS) software 20.0.

\section{RESULTS}

In total, 60 patients were enrolled in this study. The CONSORT flow diagram for patient inclusion is shown in Fig. 3. There were no differences between the two groups with regard to demographic data (Table 1).

There was a significant difference in total NTI time between the two groups (Group M, 59.7 (5.1) sec vs Group T, 52.4 (3.1) sec) (Table 2).

Mild epistaxis was observed in 6 patients in Group M and 5 patients in Group T (6/30 vs 5/30, respectively), and no moderate or severe epistaxis was observed in either group (Table 2). There was no presence of damaged oral tissue or damage to the teeth in either group. 
All NTIs were completed successfully, and no Magill forceps were used in Group T, but all were used in Group M. There was no obvious nasal pain at any time point after extubation in either group, which was similar to the result of our previous report; therefore, the data were not shown. Additionally, the sore throat in our study was not assessed because some of the surgeries were performed on the vocal cords.

\section{DISCUSSION}

Magill forceps are usually needed to facilitate insertion of the endotracheal tube into the glottis [1-3, 12, $14,15]$. However, they may cause rupture of the cuff or mucosal injury and even lead to infection[5-7]. Moreover, this instrument is always not a disposable sterilised medical device and requires strict aseptic techniques after surgery. It was reported that the percentage of needing for Magill forceps with conventional techniques was close to $70 \%$ [1]; however, it was $100 \%$ in our study. This may be because the wire-reinforced tracheal tube equipment used in our study was so soft that the tips of the tube could not be easily sent into the glottis. In our present study, the tube core, a disposable sterilised stylet, could completely replace the use of Magill forceps in NTI and decrease the NTI time significantly. This process could significantly avoid infection caused by Magill forceps and make the NTI process more convenient.

A systematic review has demonstrated that the risk of patient infection following a reusable device is significant, warranting a need for guidelines on reprocessing to be stricter to ensure greater patient safety, and indeed, when considering the risk of infection in the cost analysis, the findings from this study suggest benefits of disposable medical apparatuses in terms of cost effectiveness, cross-contamination and resource utilization [16]. Miller et al.[6] found that many cleaning methods had not removed all proteinaceous material, which showed that even following the guidelines for cleaning the equipment may be insufficient to protect patients from transmission of iatrogenic disease, although methods and techniques have been further improved. Staining was even present in $60 \%$ of the Magill forceps group. Therefore, this situation prompted us to seriously question the safety of reuse instruments. Perioperative infection has a significant impact on the outcome of surgical patients. Anaesthesiologists have roles in reducing infection by applying appropriate prophylactic measures [17]. Magill forceps are not always disposable sterilised medical devices and require strict aseptic techniques after surgery. Perhaps we could consider the tube core, a sterile stylet, as an optimal choice in NTI where possible. Of course, further study through a large sample is needed to confirm the effectiveness.

It was reported that the wire tube body of the Disposcope endoscope was rigid but could be bent along the radian of the nasal cavity, which benefitted NTI [9]. Another device, a video intubating stylet for NTI, which has a rigid intubating stylet with an adjustable distal portion, makes the process of NTI quicker and easier[18]. In those studies, the tube stylet was advanced through into the glottis easily by their levelling effect but did not increase the incidence of related complications $[9,14,18]$. Although the tube core has a malleable rigid body, it protects the nasal mucosa and the entire nasal passage during the operational process by wire-reinforced tracheal tube, with results similar to those in our previous study [9].

Epistaxis, or postpharyngeal bleeding, is the most common complication after NTI. Thermosoftening of the endotracheal tube should not be overlooked even if other effective methods, such as telescoping the endotracheal tube into a rubber catheter, have already been applied because it has the obvious advantages of reducing the incidence of epistaxis and improving the nasal passage of the endotracheal tube [3, 15]. The wire-reinforced tracheal tube in our study is a soft endotracheal tube that contributes to reducing bleeding[9]. Therefore, simple thermosoftening treatment of the endotracheal tube has not been performed in the present research. On the other hand, NTI under suction catheter guidance increases the success rate of airway instrumentation and reduces the incidence and severity of epistaxis [19]. All of these factors may have decreased the severity of epistaxis in our study.

To be sure, there are some limitations to our study. First, the operator was not blinded to the research, which may have affected the process of NTI. Second, unfortunately, the risk of infection had not be assessed through a large sample of research, which would provide guidance for clinical treatment. Third, patients with preoperative modified Mallampati scores of III or IV were excluded from our study, which should be 
addressed in our research in the future. Furthermore, the NTI was completed under a Macintosh laryngoscope in the present study. However, videolaryngoscopy is associated with a significantly decreased force exerted on maxillary incisors and might reduce the risk for dental injury in clinical settings [20]; moreover, videolaryngoscopy can clearly observe the glottis, which can simplify the process of NTI.

\section{CONCLUSIONS}

This study shows that using a tube core, a disposable sterilised stylet, could completely replace the use of Magill forceps in NTI and reduce the NTI time significantly without a difficult airway. Therefore, using a tube core for NTI without a difficult airway may be a convenient choice when possible.

\section{ACKNOWLEDGEMENTS}

The authors would like to thank all the staff of the Department of Anaesthesiology, The Third Affiliated Hospital of Anhui Medical University (The First People's Hospital of Hefei) and the Department of Otorhinolaryngology Surgery, The Third Affiliated Hospital of Anhui Medical University (The First People's Hospital of Hefei) for their help in finishing this research.

\section{AUTHOR CONTRIBUTIONS}

R. Hu and J.Y. Niu helped design the study, conduct the study, collect the data, analyse the data, and write the manuscript. L.N Wu, H. Sun and P. Sun helped to conduct the study and collect the data. J.Y Huang helped to conduct the study. J.M. Yu helped to design the study and write the manuscript. All authors have read and approved the manuscript submitted for publication.

\section{FINANCIAL SUPPORT}

No funding was obtained for this study.

\section{REFERENCES}

1. Lim CW, Min SW, Kim CS, Chang JE, Park JE, Hwang JY. The use of a nasogastric tube to facilitate nasotracheal intubation: a randomised controlled trial. Anaesthesia. 2014;69(6):591-597.

2. Pourfakhr P, Ahangari A, Etezadi F, et al. Comparison of Nasal Intubations by GlideScope With and Without a Bougie Guide in Patients Who Underwent Maxillofacial Surgeries: Randomized Clinical Trial. Anesthesia and analgesia. 2018;126(5):1641-1645.

3. Kim EM, Chung MH, Lee MH, et al. Is Tube Thermosoftening Helpful for Videolaryngoscope-Guided Nasotracheal Intubation?: A Randomized Controlled Trial. Anesthesia and analgesia. 2019;129(3):812-818.

4. Abrons RO, Zimmerman MB, El-Hattab YMS. Nasotracheal intubation over a bougie vs. non-bougie intubation: a prospective randomised, controlled trial in older children and adults using videolaryngoscopy. Anaesthesia. 2017;72(12):1491-1500.

5. Nakamura S, Watanabe T, Hiroi E, Sasaki T, Matsumoto N, Hori T. Cuff damage during naso-tracheal intubation for general anesthesia in oral surgery. Masui. 1997;46(11):1508-1514.

6. Miller DM, Youkhana I, Karunaratne WU, Pearce A. Presence of protein deposits on 'cleaned' re-usable anaesthetic equipment. Anaesthesia. 2001;56(11):1069-1072.

7. Mahajan R, Batra YK, Kumar S. Another use of Magill forceps to assist nasotracheal intubation. Can J Anaesth. 2007;54(11):957-958.

8. Kumar R, Gupta E, Kumar S, Rani Sharma K, Rani Gupta N. Cuff inflation-supplemented laryngoscope-guided nasal intubation: a comparison of three endotracheal tubes. Anesthesia and analgesia. 2013;116(3):619-624.

9. Yu J, Hu R, Wu L, Sun P, Zhang Z. A comparison between the Disposcope endoscope and fibreoptic bronchoscope for nasotracheal intubation: a randomized controlled trial. BMC Anesthesiol. 2019;19(1):163. 
10. Driver BE, Prekker ME, Klein LR, et al. Effect of Use of a Bougie vs Endotracheal Tube and Stylet on First-Attempt Intubation Success Among Patients With Difficult Airways Undergoing Emergency Intubation: A Randomized Clinical Trial. JAMA. 2018;319(21):2179-2189.

11. Cormack RS, Lehane J. Difficult tracheal intubation in obstetrics. Anaesthesia. 1984;39(11):1105-1111.

12. Yeom JH, Oh MK, Shin WJ, Ahn DW, Jeon WJ, Cho SY. Randomized comparison of the effectiveness of nasal intubation using a GlideScope video laryngoscope with Magill forceps versus vascular forceps in patients with a normal airway. Can J Anaesth. 2017;64(12):1176-1181.

13. Earle R, Shanahan E, Vaghadia H, Sawka A, Tang R. Epistaxis during nasotracheal intubation: a randomized trial of the Parker Flex-Tip nasal endotracheal tube with a posterior facing bevel versus a standard nasal RAE endotracheal tube. Can J Anaesth. 2017;64(4):370-375.

14. Staar S, Biesler I, Muller D, Pfortner R, Mohr C, Groeben H. Nasotracheal intubation with three indirect laryngoscopes assisted by standard or modified Magill forceps. Anaesthesia. 2013;68(5):467-471.

15. Kim YC, Lee SH, Noh GJ, et al. Thermosoftening treatment of the nasotracheal tube before intubation can reduce epistaxis and nasal damage. Anesthesia and analgesia. 2000;91(3):698-701.

16. Mouritsen JM, Ehlers L, Kovaleva J, Ahmad I, El-Boghdadly K. A systematic review and cost effectiveness analysis of reusable vs. single-use flexible bronchoscopes. Anaesthesia. 2020;75(4): 529-540.

17. Shime N. Role of anesthesiologist in prevention of perioperative infection. Masui. 2014;63(3):269-277.

18. Lee MC, Tseng KY, Shen YC, et al. Nasotracheal intubation in patients with limited mouth opening: a comparison between fibreoptic intubation and the Trachway(R). Anaesthesia. 2016;71(1):31-38.

19. Morimoto Y, Sugimura M, Hirose Y, Taki K, Niwa H. Nasotracheal intubation under curve-tipped suction catheter guidance reduces epistaxis. Can J Anaesth. 2006;53(3):295-298.

20. Schieren M, Kleinschmidt J, Schmutz A, et al. Comparison of forces acting on maxillary incisors during tracheal intubation with different laryngoscopy techniques: a blinded manikin study. Anaesthesia. 2019;74(12):1563-1571.
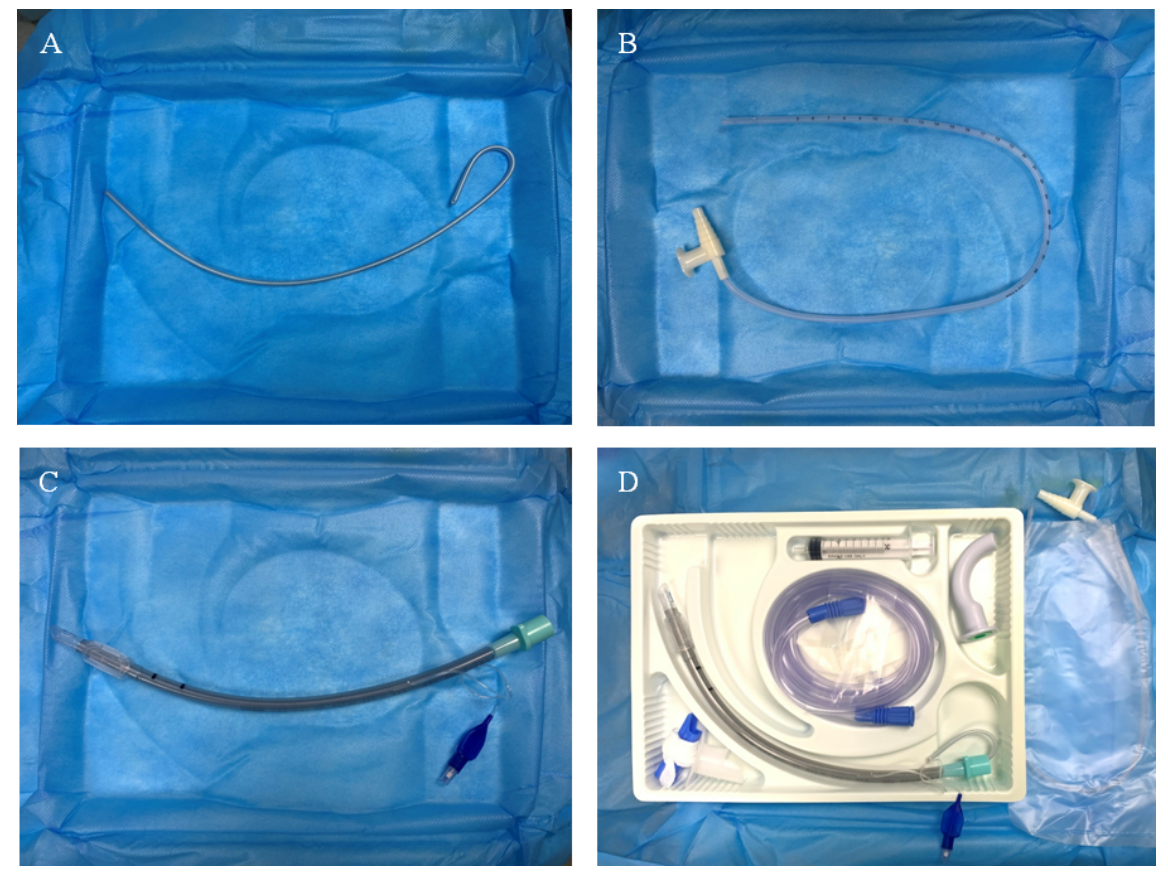
A

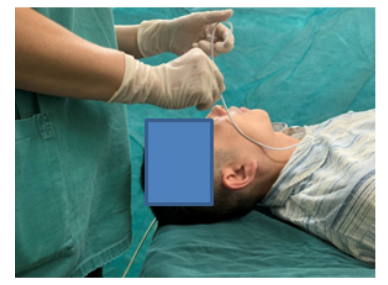

$\mathrm{D}$

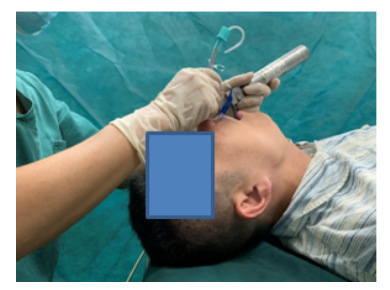

B

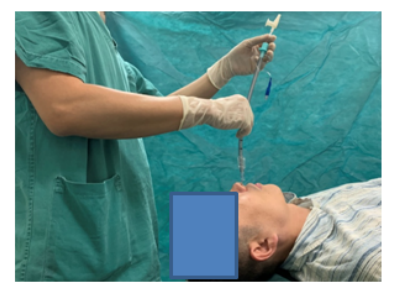

E

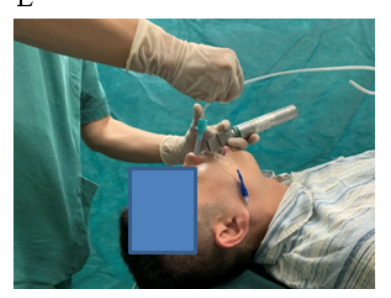

C

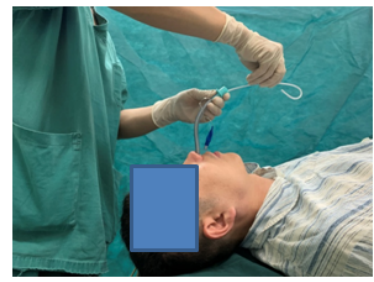

$\mathrm{F}$

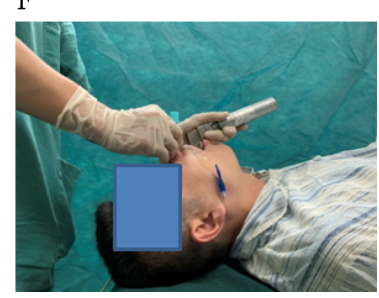

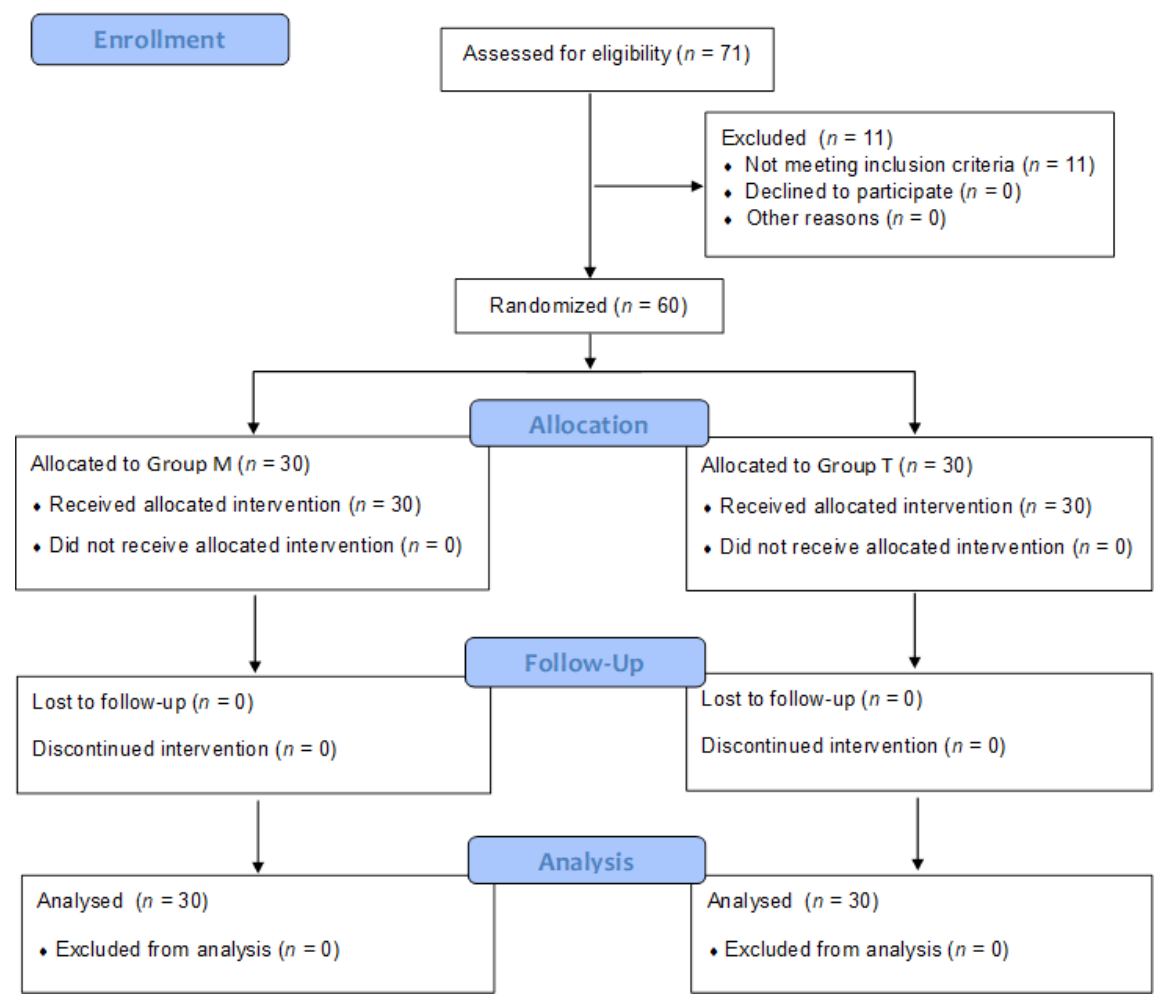

\section{Hosted file}

Tables.pdf available at https://authorea.com/users/378929/articles/495324-comparison-withtube-core-and-magill-forceps-for-nasotracheal-intubation-a-randomized-controlled-trial 